\title{
Effect of Thermal Shrinkage of Extruded Sheet on Mouthguard Thickness: Influence of Model Undercut
}

\author{
Mutsumi Takahashi ${ }^{*}$, Yogetsu Bando² \\ ${ }^{1}$ Department of Physiology, The Nippon Dental University School of Life Dentistry at Niigata, Niigata, Japan \\ ${ }^{2}$ BANDO Dental Clinic, Ishikawa, Japan \\ Email: *mutsumit@ngt.ndu.ac.jp
}

How to cite this paper: Takahashi, M. and Bando, Y. (2022) Effect of Thermal Shrinkage of Extruded Sheet on Mouthguard Thickness: Influence of Model Undercut. Materials Sciences and Applications, 13, 54-62. https://doi.org/10.4236/msa.2022.131004

Received: December 23, 2021

Accepted: January 26, 2022

Published: January 29, 2022

Copyright (๑) 2022 by author(s) and Scientific Research Publishing Inc. This work is licensed under the Creative Commons Attribution International License (CC BY 4.0).

http://creativecommons.org/licenses/by/4.0/ (c) (i) Open Access

\begin{abstract}
The effectiveness and safety of the mouthguard are greatly affected by its thickness. The aim of this study was to investigate the effect of thermal shrinkage of the extruded sheet on the mouthguard thickness depending on the amount of undercut of the model. Mouthguard sheet was used a $4.0 \mathrm{~mm}$ thick ethylene-vinyl acetate resin manufactured by extrusion molding. The sheets were placed in the vacuum forming machine with the sheet extrusion direction either vertical (condition $\mathrm{V}$ ) or parallel (condition $\mathrm{P}$ ) to the model's centerline. The working models were three hard plaster models trimmed so that the angles of the anterior teeth to the model base were $90^{\circ}, 100^{\circ}$, and $110^{\circ}$ (Models $\mathrm{A}, \mathrm{B}$, and C). The sheet was softened until it sagged $15 \mathrm{~mm}$, and then suction was continued for $30 \mathrm{~s}$. Measurement points of the mouthguard were the incisal portion (incisal edge and labial surface) and molar portion (cusp and buccal surface). The differences in the reduction rate of the thickness due to model form and extrusion direction were analyzed using two-way ANOVA and Bonferroni's multiple comparison tests. Differences in thickness depending on the extrusion direction of the sheet were observed in Models B and C on the labial surface and in all models on the buccal surface, and the thicknesses obtained under condition $\mathrm{P}$ were significantly thinner than those obtained under condition $\mathrm{V}$. The thicknesses of the incisal edge and the cusp were not affected by the extrusion direction. The result of this study was suggested that the labial and buccal thickness of the mouthguard was secured by placing the sheet in the extrusion direction vertical to the model's centerline. Furthermore, it was clarified that the presence of the undercut of the model tends to increase the influence of the extrusion direction of the sheet on the thickness of the mouthguard.
\end{abstract}




\section{Keywords}

Mouthguard, Extrusion Molding, Thickness, Model Angle, Undercut

\section{Introduction}

The effectiveness and safety of mouthguards depend on the material and thickness of the sheet [1] [2] [3]. To counter the stress and strain generated during impacts, it is necessary to provide a sufficient thickness of $3-4 \mathrm{~mm}$ on the labial and buccal sides of the mouthguard [4] [5]. For single-sheet mouthguards, a 4.0mm-thick sheet is often used. However, because the thickness is greatly reduced by thermoforming, it is difficult to secure the thickness required for impact absorption. Laminated mouthguards reliably provide a suitable thickness, but they may not be readily available to users due to the cost and fabricating time. Therefore, various fabrication methods have been investigated to secure the thickness after formation with a single sheet [6] [7] [8].

Mouthguard sheets are manufactured by extrusion or injection molding. Most of the sheets of ethylene-vinyl acetate resin (EVA), which is a mainstream product currently on the market, are manufactured by extrusion molding. Extruded sheets shrink in the extrusion direction as the strain introduced during manufacturing is released during thermoforming. This thermal shrinkage affects the mouthguard thickness depending on the sheet and model installation direction. In contrast, sheets manufactured by injection molding are not distorted during sheet manufacturing, and thus do not undergo thermal shrinkage during thermoforming [9]. Previously, the effect of the heating conditions and the shape of the sheet on the thermal shrinkage that occurs in the extruded sheet have been investigated [7].

There have been studies on the effect of model angles on the mouthguard thickness [10] [11]. These findings indicate that the presence of labial undercuts extends the mouthguard sheet and reduces labial thickness. For athletes with maxillary anterior teeth tilted to the labial side, trimming the model so that there is no undercut on the labial side tends to increase the model height. However, as the model height increases, the mouthguard becomes thinner [4] [12]. Athletes with a large overjet have a higher risk and severity of sports injuries [13] [14], indicating that the mouthguard thickness is particularly important for this type of model form. The present study was envisioned the fabrication of a mouthguard for athletes with maxillary anterior teeth tilted labially. Therefore, it was verified how much the amount of undercut in the model affected thermal shrinkage. The null hypothesis was that the thermal shrinkage of the sheet was not affected by the model form.

\section{Materials and Methods}

The working model was fabricated using a silicone rubber (Correcsil, Yamahachi Dental Mfg., Co., Aichi, Japan) impression taken from a maxillary dental model 
(D16FE-500A-QF, Nissin Dental Products Inc., Kyoto, Japan), into which dental gypsum (New Plastone, GC, Co., Tokyo, Japan) was poured [6] [7]. The plaster model was trimmed into the following forms using a model trimmer (MT-6, Morita, Co., Tokyo, Japan): 1) Model A, in which the angle of the model formed between the labial surface of the central incisor and the base of the working model was $90^{\circ}$ with a height of $25 \mathrm{~mm}$ at the incisal edge of the maxillary central incisor and a height of $20 \mathrm{~mm}$ at the mesiobuccal cusp of the maxillary first molar (i.e., undercut amount on the labial side $0^{\circ}$ ); 2) Model B, in which the angle was $10^{\circ}$ greater than in Model A with a height of $25 \mathrm{~mm}$ at the incisal edge of the maxillary central incisor and a height of $25 \mathrm{~mm}$ at the mesiobuccal cusp of the maxillary first molar (i.e., undercut amount on the labial side $10^{\circ}$ ); and 3) Model C, in which the angle was $20^{\circ}$ greater than in Model A with a height of $25 \mathrm{~mm}$ at the incisal edge of the maxillary central incisor and a height of $30 \mathrm{~mm}$ at the mesiobuccal cusp of the maxillary first molar (i.e., undercut amount on the labial side $20^{\circ}$ ) (Figure 1).

(a)

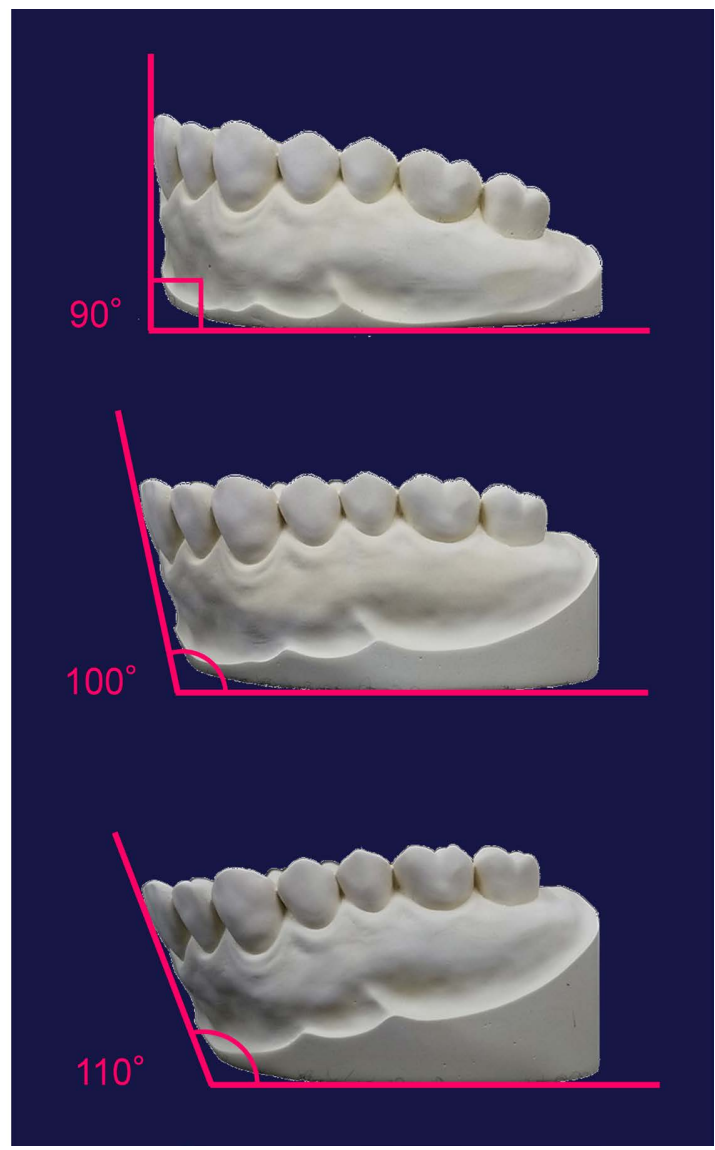

Figure 1. Working models. Angles formed between the labial surface of the central incisor and the base of the working model of (a), $90^{\circ}$ (Model A; height of $25 \mathrm{~mm}$ at the incisal edge of the maxillary central incisor and $20 \mathrm{~mm}$ at the mesiobuccal cusp of the maxillary first molar); (b) $100^{\circ}$ (Model B; height of $25 \mathrm{~mm}$ at the incisal edge of the maxillary central incisor and $25 \mathrm{~mm}$ at the mesiobuccal cusp of the maxillary first molar); (c) $110^{\circ}$ (Model (c); height of $25 \mathrm{~mm}$ at the incisal edge of the maxillary central incisor and $30 \mathrm{~mm}$ at the mesiobuccal cusp of the maxillary first molar). 
Mouthguards were thermoformed using EVA sheets (Sports Mouthguard, Keystone Dental Inc., Cherry Hill, NJ; $127 \times 127 \times 4.0 \mathrm{~mm}$, clear) and a vacuum forming machine (Pro-form, T \& S Dental \& Plastics Co., Inc., Myerstown, PA). The sheet was mounted on the forming machine so that the sheet extrusion direction was vertical (condition $\mathrm{V}$ ) or parallel (condition $\mathrm{P}$ ) to the model's centerline. The model position was $25 \mathrm{~mm}$ from the front of the forming unit. The sheet was softened until it sagged $15 \mathrm{~mm}$, and then suction was continued for 30 $\mathrm{s}$ [6] [7]. The model was left in place for at least $24 \mathrm{~h}$ before the mouthguard was removed. Six specimens were formed for each condition; thus, a total of 36 mouthguards were fabricated (i.e., 2 extrusion direction $\times 3$ model forms $\times 6$ repetitions).

Mouthguard thickness was measured using a specialized caliper accurate to $0.1 \mathrm{~mm}$ (21-111, YDM, Co., Tokyo, Japan) without a spring, so as to prevent distortion during measurement [6] [7]. The measurement points were the left and right central incisors ( 10 points on the incisal edge and 20 points on the labial surface) and the first molars (8 points on the cusp and 20 points on the buccal surface) [6] [7]. The measurements were taken once for each specimen.

The differences in the reduction rate of the thickness due to model form and extrusion direction were analyzed using statistical analysis software (IBM SPSS 24.0, SPSS Japan Inc., Tokyo, Japan). The Shapiro-Wilk test for normality of distribution and Levene's test for homogeneity of variance were also used. Each measurement exhibited normality and equal dispersion; accordingly, analysis was performed by two-way analysis of variance (ANOVA) and Bonferroni's multiple comparison tests. All analytical methods were performed with a significance level of $5 \%$ and a detection power of $80 \%$, and the difference was considered significant when both were satisfied.

\section{Results and Discussion}

Table 1 shows the results of two-way ANOVA for the mouthguard thickness after formation. At all measurement points, the main effects of the model form and extraction direction were significant, and their interaction was also significant. Based on the results, simple main effect tests were performed prior to multiple comparisons among levels.

Table 2 and Figure 2 show the results of multiple comparison analysis of the mouthguard thickness reduction. At all measurement sites, the rate of decrease in thickness tended to increase as the model angle increased. Differences in thickness depending on the extrusion direction of the sheet were observed in Models $\mathrm{B}$ and $\mathrm{C}$ on the labial surface and in all models on the buccal surface, and the thicknesses obtained under condition P were significantly thinner than those obtained under condition $\mathrm{V}$. The thickness of the incisal edge and the cusp was not affected by the extrusion direction.

Thermal shrinkage of the mouthguard sheet occurs when the strain accumulated during manufacturing is released from the extruded sheet [9]. Because 
Table 1. Results of two-way ANOVA for thickness after formation.

\begin{tabular}{lccccc}
\hline Source & $\boldsymbol{d f}$ & SS & MS & $F$-value & $P$-value \\
\hline Incisal edge & & & & & \\
$\quad$ Model form (A) & 2 & 108.431 & 54.215 & 671.166 & $<0.001^{\star *}$ \\
$\quad$ Extrusion direction (B) & 1 & 53.290 & 53.290 & 659.711 & $<0.001^{* *}$ \\
$\mathrm{~A}^{\star \mathrm{B}}$ & 2 & 9.912 & 4.956 & 61.351 & $<0.001^{* *}$ \\
Error & 30 & 2.423 & 0.081 & &
\end{tabular}

Labial surface

$\begin{array}{llllll}\text { Model form (A) } & 2 & 291.527 & 145.764 & 2342.629 & <0.001^{\star *} \\ \text { Extrusion direction (B) } & 1 & 30.250 & 30.250 & 486.161 & <0.001^{\star \star} \\ \mathrm{A}^{\star \mathrm{B}} & 2 & 2.062 & 1.031 & 16.567 & <0.001^{\star \star} \\ \text { Error } & 30 & 1.867 & 0.062 & & \end{array}$

\section{Cusp}

$\begin{array}{lccccc}\text { Model form (A) } & 2 & 96.616 & 48.308 & 649.880 & <0.001^{\star *} \\ \text { Extrusion direction (B) } & 1 & 0.640 & 0.640 & 8.610 & <0.001^{\star *} \\ \mathrm{~A}^{\star} \mathrm{B} & 2 & 1.807 & 0.903 & 12.152 & <0.001^{\star *} \\ \text { Error } & 30 & 2.230 & 0.074 & & \end{array}$

\section{Buccal surface}

$\begin{array}{lccccc}\text { Model form (A) } & 2 & 357.717 & 178.859 & 1719.794 & <0.001^{\star \star} \\ \text { Extrusion direction (B) } & 1 & 52.321 & 52.321 & 503.088 & <0.001^{\star \star} \\ \mathrm{A}^{\star \mathrm{B}} & 2 & 8.101 & 4.050 & 38.945 & <0.001^{\star \star} \\ \text { Error } & 30 & 3.120 & 0.104 & & \end{array}$

$d f$ degree of freedom; SS: sum of squares; MS: mean square; ${ }^{* *} P<0.01$ : denotes statistically significant difference.

Table 2. Results of Bonferroni's multiple comparison test according to model form.

Incisal edge
\begin{tabular}{|c|c|c|c|}
\hline Condition V & Model A & Model B & Model C \\
\hline Model A & & & \\
\hline Model B & $*$ & & \\
\hline Model C & $* *$ & n.s. & \\
\hline
\end{tabular}

Cusp

\begin{tabular}{|c|c|c|c|}
\hline Condition V & Model A & Model B & Model C \\
\hline Model A & & & \\
\hline Model B & n.s. & & \\
\hline Model C & $* *$ & $* *$ & \\
\hline
\end{tabular}

\begin{tabular}{|c|c|c|c|}
\hline Condition P & Model A & Model B & Model C \\
\hline Model A & & & \\
\hline Model B & ${ }^{*}$ & & \\
\hline Model C & ${ }^{* *}$ & n.s. & \\
\hline \multicolumn{4}{|c|}{${ }^{* *} P<0.01 ;{ }^{*} P<0.05$; n.s.: not significant. }
\end{tabular}

\begin{tabular}{|c|c|c|c|}
\hline Condition P & Model A & Model B & Model C \\
\hline Model A & & & \\
\hline Model B & n.s. & & \\
\hline Model C & $* *$ & $* \star$ & \\
\hline \multicolumn{4}{|c|}{${ }^{* *} P<0.01$; n.s.: not significant. }
\end{tabular}


Labial surface

\begin{tabular}{|c|c|c|c|}
\hline Condition V & Model A & Model B & Model C \\
\hline Model A & & & \\
\hline Model B & $* *$ & & \\
\hline Model C & $* *$ & $*$ & \\
\hline
\end{tabular}

\begin{tabular}{|c|c|c|c|}
\hline Condition P & Model A & Model B & Model C \\
\hline Model A & & & \\
\hline Model B & $* *$ & & \\
\hline Model C & $* \star$ & $* *$ & \\
\hline \multicolumn{4}{|c|}{${ }^{* *} P<0.01 \cdot{ }^{*} P<0.05$} \\
\hline
\end{tabular}

(a)

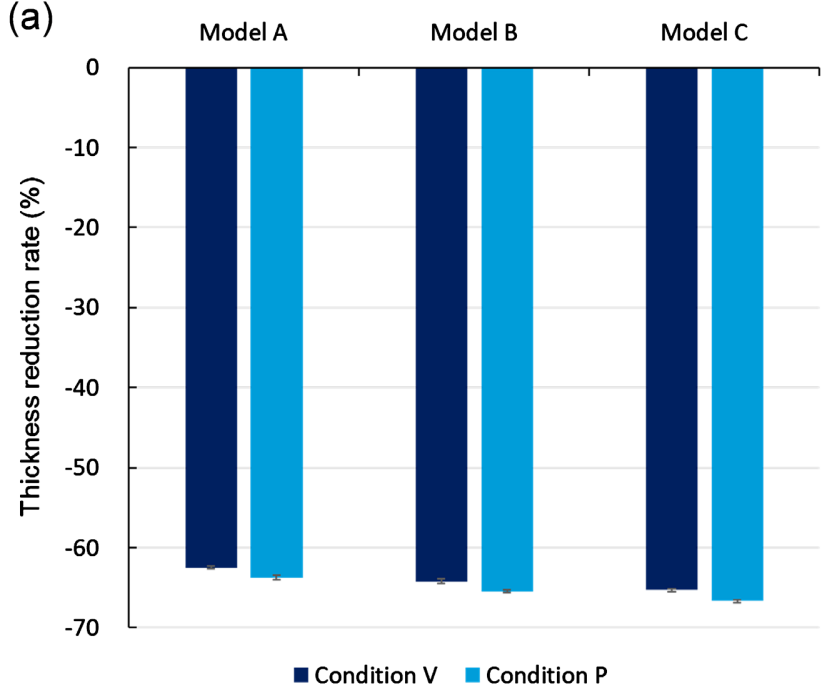

(b)

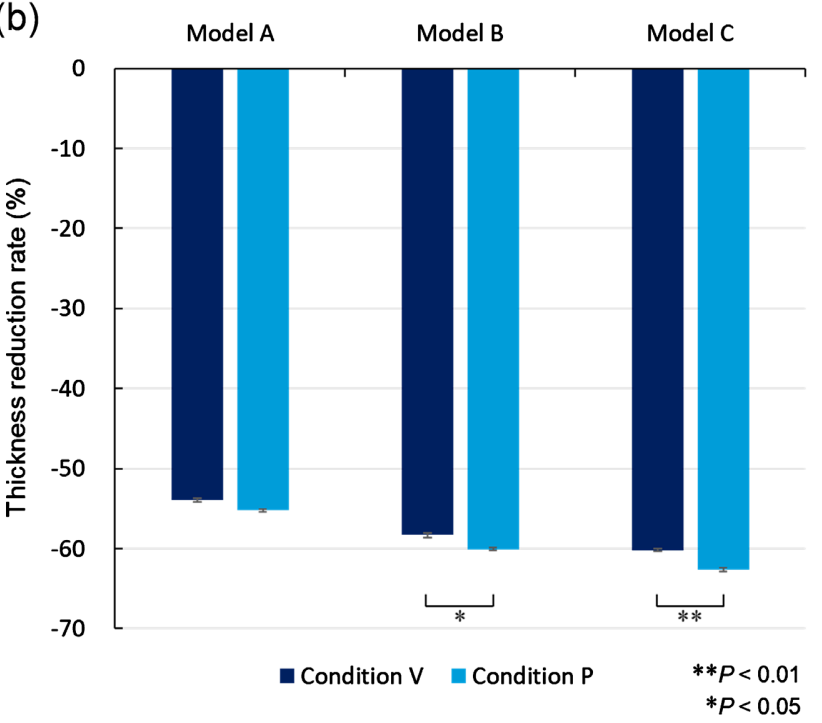

(d)
Buccal surface

\begin{tabular}{|c|c|c|c|}
\hline Condition V & Model A & Model B & Model C \\
\hline Model A & & & \\
\hline Model B & $* *$ & & \\
\hline Model C & $* *$ & $* *$ & \\
\hline
\end{tabular}

\begin{tabular}{|c|c|c|c|}
\hline Condition P & Model A & Model B & Model C \\
\hline Model A & & & \\
\hline Model B & $* *$ & & \\
\hline Model C & $* *$ & $* *$ & \\
\hline \multicolumn{4}{|c|}{${ }^{* *} P<0.01$} \\
\hline
\end{tabular}

(c)
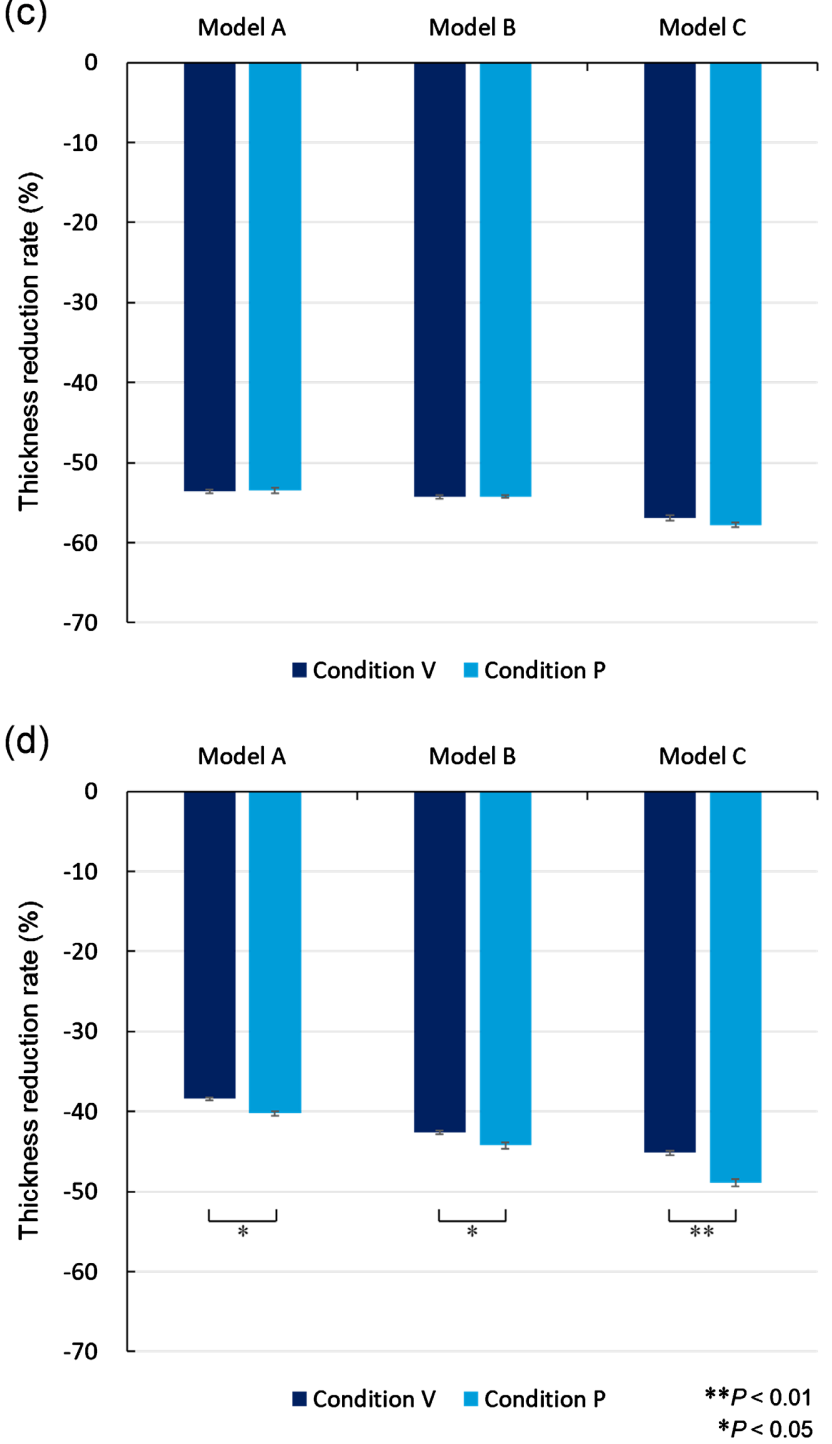

Figure 2. Mouthguard thickness at measurement points on: (a) the incisal edge; (b) the labial surface; (c) the cusp; and (d) the buccal surface. Measurements are expressed as mean value \pm SD. 
thermal shrinkage occurs along the orientation direction of the sheet, the sheet shrinks in the extrusion direction. When the softened sheet is pressed against the model, the sheet is affected by the model form and has a part that extends and a part that shrinks, and thus a non-uniform shape change occurs [9] [15]. It has been reported that the mouthguard thickness is affected by factors including the model shape, the sheet material and color, and the heating conditions of the sheet [4] [11] [12] [16] [17]. However, the extent of the effect of thermal shrinkage due to the extrusion direction of the sheet on the mouthguard thickness has not been clarified for different model forms. The model form in this study was based on Model $\mathrm{A}$, which has no undercut on the labial side and the height is reduced as much as possible [6] [11] [15] [17]. Models B and C were prepared by adjusting the height of the molars so that the central incisor tooth axis was tilted $10^{\circ}$ and $20^{\circ}$ more than Model A, respectively, and the amount of undercut on the labial side was increased.

As a result of this study, the effect of thermal shrinkage of the sheet on the mouthguard thickness differed depending on the amount of the model undercut. Therefore, the null hypothesis was rejected.

The thickness of the mouthguard on the anterior part tended to decrease as the model angle (i.e., the amount of undercut) increased. This is consistent with previous studies [10] [11] showing that changes in model angle affect thickness. During model pressure welding, the sheet is fixed by the part in contact with the incisal edge and the part held by the sheet frame is stretched in the direction of the model immediately by subsequent suction or pressurization [9]. That is, if there is an undercut, the stretching in the direction of the model is large, and the mouthguard labial side tends to be thin [9]. At the incisal edge, there was no significant difference depending on the extrusion direction of the sheet, but condition $\mathrm{P}$ tended to increase the rate of decrease in thickness. Under condition $\mathrm{P}$, the labial surface was significantly thinner in Models B and C. Because the anterior-posterior width of the model anterior part is narrow, the softened sheet can stretch greatly in the anterior-posterior direction during pressure welding [18]. The direction perpendicular to the extrusion direction has a smaller effect on the thickness of the material resin than that of parallel [19]; thus, the thickness obtained under condition $\mathrm{P}$ was smaller than that obtained under condition $\mathrm{V}$.

The molar thickness of the mouthguard tends to decrease as the model angle increases. The model in this study had a morphology in which the molars became higher as the angle increased, similar to previous studies [4] [12], in which the mouthguard became thinner as the model height increased. In the present study, as the height of the model increased, the effect of the extrusion direction of the sheet on the buccal thickness of the mouthguard increased. The molar part of the model has a wide lateral width in addition to the anterior-posterior width, and thus the softened sheet stretches in the left-right direction as well as in the anterior-posterior direction during pressure welding [18]. The direction perpendicular to the extrusion direction has a small effect on the thickness of the 
material resin, whereas the direction parallel to the extrusion direction has a large effect on the thickness [19]. Therefore, it was inferred that the decrease in thickness due to a slight extension was larger under condition $\mathrm{P}$ than condition $\mathrm{V}$, similar to the anterior part.

\section{Conclusion}

The form of the working model, especially the presence of undercuts on the labial side, affects the labial thickness of the mouthguard during thermoforming. In cases where the height of the model increases by trimming to reduce the amount of undercut (i.e., the labial side of the anterior teeth of the upper jaw is tilted), it was clarified that it is effective in suppressing the decrease in the labial and buccal thicknesses of the mouthguard by placing the sheet in the extrusion direction perpendicular to the model's centerline. However, this method did not secure the mouthguard thickness required for shock absorption. To maintain an appropriate thickness, it is essential to devise a forming method and laminated mouthguards will be considered in future work.

\section{Conflicts of Interest}

The authors declare no conflicts of interest regarding the publication of this paper.

\section{References}

[1] Bochnig, M.S., Oh, M.J., Nagel, T., Ziegler, F. and Jost-Brinkmann, P.G. (2017) Comparison of the Shock Absorption Capacities of Different Mouthguards. Dental Traumatology, 33, 205-213. https://doi.org/10.1111/edt.12324

[2] Gawlak, D., Mańka-Malara, K., Mierzwińska-Nastalska, E., Gieleta, R., Kamiński, T. and Łuniewska, M. (2017) A Comparison of Impact Force Reduction by Polymer Materials Used for Mouthguard Fabrication. Acta Bioeng Biomech, 19, 89-95.

[3] Tribst, J.P.M., de Oliveira, D.P.A.M., Borges, A.L.S. and Bottino, M.A. (2018) Influence of Custom-Made and Stock Mouthguard Thickness on Biomechanical Response to a Simulated Impact. Dental Traumatology, 34, 429-437. https://doi.org/10.1111/edt.12432

[4] Geary, J.L. and Kinirons, M.J. (2008) Post Thermoforming Dimensional Changes of Ethylene Vinyl Acetate Used in Custom-Made Mouthguards for Trauma Prevention: A Pilot Study. Dental Traumatology, 24, 350-355. https://doi.org/10.1111/j.1600-9657.2007.00550.x

[5] Maeda, M., Takeda, T., Nakajima, K., Shibusawa, M., Kurokawa, K. and Shimada, A. (2008) In Search of Necessary Mouthguard Thickness: Part 1 from the View Point of Shock Absorption Ability. Journal of Prosthodontic Research, 52, 211-219. https://doi.org/10.2186/jips.52.211

[6] Takahashi, M. and Bando, Y. (2019) Thermoforming Method to Effectively Maintain Mouthguard Thickness: Effect of Moving the Model Position Just before Vacuum Formation. Dental Traumatology, 35, 121-127.

https://doi.org/10.1111/edt.12447

[7] Takahashi, M. and Koide, K. (2016) Variation in Mouthguard Thickness According to Heating Conditions during Fabrication: Part 2 Sheet Shape and Effect of Thermal 
Shrinkage. Dental Traumatology, 32, 185-191. https://doi.org/10.1111/edt.12209

[8] Farrington, T., Coward, T., Onambele-Pearson, G., Taylor, R.L., Earl, P. and Winwood, K. (2016) An Investigation into the Relationship between Thickness Variations and Manufacturing Techniques of Mouthguards. Dental Traumatology, 32, 14-21. https://doi.org/10.1111/edt.12192

[9] Takeuchi, M. and Togaya, N. (2006) Effectively of Thermoforming Process for Fabricating of Intraoral Apparatus. Sunashobo, Tokyo, 21-26, 33-44, 54-55, 62-67, 81. (in Japanese)

[10] Farrington, T., Coward, T., Onambele-Pearson, G., Taylor, R.L., Earl, P. and Winwood, K. (2016) The Effect of Model Inclination during Fabrication on Mouthguard Calliper-Measured and CT Scan-Assessed Thickness. Dental Traumatology, 32, 192-200. https://doi.org/10.1111/edt.12213

[11] Takahashi, M. and Bando, Y. (2021) Fabrication Method to Maintain Mouthguard Thickness Regardless of the Model Angle. Dental Traumatology, 37, 131-137. https://doi.org/10.1111/edt.12584

[12] Del, R.G. and Leyte, V.M.A. (2007) Fabricating a Better Mouthguard: Part I Factors Influencing Mouthguard Thinning. Dental Traumatology, 23, 149-154.

https://doi.org/10.1111/j.1600-9657.2006.00436.x

[13] Patel, M.C. and Sujan, S.G. (2012) The Prevalence of Traumatic Dental Injuries to Permanent Anterior Teeth and Its Relation with Predisposing Risk Factors among 8-13 Years School Children of Vadodara City: An Epidemiological Study. Journal of the Indian Society of Pedodontics and Preventive Dentistry, 30, 151-157. https://doi.org/10.4103/0970-4388.99992

[14] Ain, T.S., Telgi, R.L., Sultan, S., Tangade, P., Telgi, C.R., Tirth, A., et al. (2016) Prevalence of Traumatic Dental Injuries to Anterior Teeth of 12-Year-Old School Children in Kashmir, India. Archives of Trauma Research, 5, Article ID: e24596. https://doi.org/10.5812/atr.24596

[15] Takahashi, M., Araie, Y., Satoh, Y. and Iwasaki, S. (2017) Shape Change in Mouthguard Sheets during Thermoforming: Part 2 Effect of the Anteroposterior Position of the Model on Mouthguard Thickness. Dental Traumatology, 33, 114-120. https://doi.org/10.1111/edt.12319

[16] Takahashi, M., Koide, K. and Mizuhashi, F. (2015) Variation in Mouthguard Thickness Due to Different Heating Conditions during Fabrication: Part 2. Dental Traumatology, 31, 18-23. https://doi.org/10.1111/edt.12122

[17] Takahashi, M. and Bando, Y. (2018) Effect of the Anteroposterior Position of the Model on Fabricated Mouthguard Thickness: Part 2 Influence of Sheet Thickness and Material. Dental Traumatology, 34, 370-377. https://doi.org/10.1111/edt.12423

[18] Takahashi, M., Kiode, K. and Mizuhashi, F. (2012) Difference in the Thickness of Mouthguards Fabricated from Ethylene-Vinyl Acetate Co-Polymer Sheets with Differently Arranged V-Shaped Grooves. Annals of Japan Prosthodontic Society, 4, 286 293. (in Japanese) https://doi.org/10.2186/ajps.4.286

[19] Takahashi, M., Koide, K., Mizuhashi, F., Mizuhashi, R., Kondo, A., Watanabe, H., et al. (2010) Influence of Thermal Shrinkage of Mouthguard Sheet Material on the Elongation and Thickness of Mouthguard Sheets after Forming. Annals of Japan Prosthodontic Society, 2, 61-69. (in Japanese) https://doi.org/10.2186/ajps.2.61 